\title{
Potential-Induced Phase Transition of Trimesic Acid Adlayer on Au(111)
}

\author{
Gui-Jin Su, ${ }^{\dagger,}+$ Hui-Min Zhang, ${ }^{\dagger}$ Li-Jun Wan, ${ }^{*}, \dagger$ Chun-Li Bai, ${ }^{*, \dagger}$ and Thomas Wandlowski ${ }^{\S}$ \\ Institute of Chemistry, Chinese Academy of Sciences (CAS), Beijing 100080, China, \\ and Research Center Jülich, Institute of Surfaces and Interfaces, ISG3, 52425 Jülich, Germany
}

Received: April 23, 2003; In Final Form: December 9, 2003

\begin{abstract}
Potential-induced phase transition of trimesic acid (TMA, $\left.\mathrm{C}_{6} \mathrm{H}_{3}(\mathrm{COOH})_{3}\right)$ on a $\mathrm{Au}(111)$ electrode was investigated by in situ scanning tunneling microscopy (STM) and cyclic voltammetry. A pair of peaks, ascribed to structural transformation was observed in the cyclic voltammograms (CVs) of $\mathrm{Au}(111)$ in $0.1 \mathrm{M} \mathrm{HClO}_{4}+$ $0.1 \mathrm{mM}$ TMA. The magnitude of the peaks decreased with increasing solution $\mathrm{pH}$. On the basis of highresolution STM images in $0.1 \mathrm{M} \mathrm{HClO}_{4}$, it is proposed that TMA molecules assume a flat-lying and a vertical orientation at 0.25 and $0.85 \mathrm{~V}$, respectively. The coexistence of the two orientations was observed at $0.65 \mathrm{~V}$. The discovered adlayer phases could be represented by $(4 \times 4),(5 \times 2 \sqrt{ } 3)$, and $(2 \sqrt{ } 3 \times 4 \sqrt{ } 3)$ or $(7 \times 2 \sqrt{ } 3)$ structures. Intriguingly, it is found that TMA molecules bind on Au substrate with one and two deprotonated carboxyl groups with potential shifting, even though the molecules take the same vertical orientation. On the basis of the STM observation, structural models for various adlayers are proposed. Comparing the STM results with those of cyclic voltammetry, we conclude that the potential-induced phase transitions in $0.1 \mathrm{M}$ $\mathrm{HClO}_{4}$ solution result from the interfacial deprotonation/protonation of the TMA molecules.
\end{abstract}

\section{Introduction}

The adsorption of organic molecules at electrode-electrolyte interfaces can be considered as one of the most promising approaches not only for the preparation of ordered adlayers but also for elucidating the nature of the electrode-electrolyte interface. ${ }^{1-5}$ Recently, the as-prepared organic monolayers were proved to be ideal models to study phase transition in lowdimensional systems, which provide a new possibility for the fabrication of nanoscale electronic devices. ${ }^{6}$ The emergence of scanning tunneling microscopy (STM), which can directly address nanoscale structures at various interfaces, ${ }^{7-12}$ has brought fascinating advances. A large number of reports have described phase transitions in organic monolayers on metal surfaces. Among these researches, nitrogen- and carboxylcontaining molecules have attracted much attention because of their importance both fundamentally and practically.

Typical nitrogen-containing molecules included pyridine, 2,2'bipyridine $\left(2,2^{\prime}-\mathrm{BP}\right)$, and uracil. Wandlowski et al. reported phase transitions in uracil adlayers on $\mathrm{Ag}, \mathrm{Au}$, and $\mathrm{Hg}$ electrode-substrate surfaces. ${ }^{13-15}$ Tao et al. reported the potential dependent order-disorder transition in a monolayer of $2,2^{\prime}-\mathrm{BP}$ on $\mathrm{Au}(111)$, which is driven by a potential dependent attractive force between the molecular chains. Further theoretical calculation shows that the substrate-mediated effective attractive force arises as an adsorbed 2,2'-BP perturbs its surrounding local surface potential and thus the nearby molecules. ${ }^{16}$ Additionally, Wan et al. reported potential-induced phase transitions of 2,2'$\mathrm{BP}$ on $\mathrm{Cu}(111) .{ }^{17}$ The reversible transition of these molecules with planar and perpendicular orientations was revealed by in situ STM. Osawa et al. studied the orientation and adlayer structure of pyridine on $\mathrm{Au}(111)$ as a function of the applied potential by using in situ surface enhanced infrared reflection

\footnotetext{
Chinese Academy of Sciences (CAS).

$\div$ Also in Graduate School of CAS, Beijing, China.

$\S$ Institute of Surfaces and Interfaces.
}

adsorption spectroscopy (SEIRAS) and STM. ${ }^{18}$ Three coordination modes of pyridine to the metal surface have been reported: (1) flat adsorption via $\pi$ electrons of the aromatic ring at negative potential; (2) tilted adsorption via both lonepair electrons of the $\mathrm{N}$ atom and $\pi$ electron as the applied potential increases; (3) N-coordinated vertical adsorption at more positive potential. The occurrence of a phase transition is rationalized as a result from a bonding transition of adsorbed pyridine.

The adsorption of carboxylic acid on metal surfaces can be considered as another model system for the study of phase transition. For instance, Zelenay et al found the adsorption of benzoic acid on polycrystalline Au not only is strong but also occurs in the entire range of potential available to electrochemical studies in aqueous solution. Direct binding of $\pi$ electrons of the benzene ring to the negatively charged surface results in parallel orientation at $E<0.30 \mathrm{~V}$, whereas a surface bond between free electron pairs on oxygens in the carboxylic group and positively charged surface results in vertical orientation at $E>1.3 \mathrm{~V}$. A region of mixed orientation cannot be ruled out in the intermediate region..$^{19}$ Thus, the phase transition occurred with the electrode potential.

In this paper, we report the potential-induced phase transition of TMA at aqueous $\mathrm{Au}(111) /$ electrolyte interfaces by in situ STM and cyclic voltammetry. The chemical structure of the molecule is shown in Chart 1. Three identical carboxyl end groups in the same plane are 3-fold-symmetrically arranged around a phenyl center showing a characteristic "propeller-like" conformation. Two additional carboxyl groups may result in different adsorption behavior for TMA, compared with the benzoic acid. On the other hand, the TMA molecule represents a prototype material for supramolecular self-assemblies. ${ }^{20}$ For TMA, the most common motif is identified as a planar honeycomb network formed by directional hydrogen bonds. ${ }^{21}$ TMA is also known to assemble in diverse supramolecular structures due to the trigonal exodentate functionality. ${ }^{22-23}$ 
CHART 1: Chemical Structure of TMA Molecule

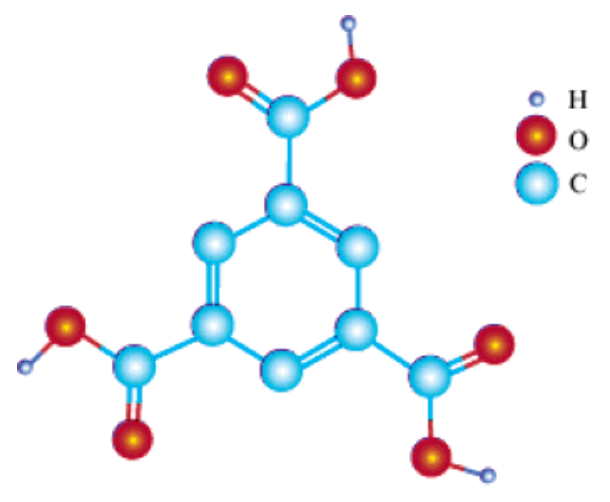

Recently, Kern et al. studied the adsorption and ordering of TMA on a $\mathrm{Cu}(100)$ surface in ultrahigh vacuum by variabletemperature scanning tunneling microscopy. ${ }^{24}$ At low temperature $(\sim 200 \mathrm{~K})$, two-dimensional networks of planar oriented molecules, interconnected by directional hydrogen bonds, were found. A rather similar structural motif was also reported in the bulk TMA crystal structure. At room temperature, more densely packed stripe arrangements exist, which were attributed to stacking rows of perpendicularly oriented molecules coordinated to the substrate via a carboxylate group. Kunitake et al. reported adsorption of TMA on $\mathrm{Au}(111)$ surface. ${ }^{25}$ A twodimensional molecular network was observed in a limited potential region. The present paper reports the adsorption of TMA adlayers in a wide potential region. A potential-induced phase transition is observed. The experiment is conducted under the aqueous conditions. Thus, it is convenient to control the molecular orientations compared with that under the ultrahigh vacuum.

\section{Experimental Section}

The STM experiments were carried out with a Nanoscope E (Digital Instruments). The tunneling tips used were prepared by electrochemical etching of a tungsten wire $(0.25 \mathrm{~mm}$ in diameter), subsequently coated with clear nail polish to minimize Faradaic currents. All STM images were acquired in constantcurrent mode. The electrodes $(\mathrm{Au}(111))$ were prepared with the bead method by melting a Au wire $(99.999 \%$ in purity, $0.8 \mathrm{~mm}$ in diameter). Before each experiment, the Au bead was annealed in a hydrogen-oxygen flame and quenched after careful cooling in Milli-Q water, which was saturated with hydrogen. ${ }^{26}$ All solutions were prepared with ultrapure Milli-Q water. Solutions of variable $\mathrm{pH}$ were prepared by mixing $0.1 \mathrm{M} \mathrm{HClO}_{4}$ and 0.1 $\mathrm{M} \mathrm{NaOH}$. Trimesic acid is obtained from Kanto Chemical Co. (Tokyo) and used without further purification. The cyclic voltammograms of $\mathrm{Au}(111)$ in various solutions were measured with an EG\&G basic electrochemical system. The solutions were deaerated with nitrogen prior to the measurements. The reference and counter electrode were a RHE and a Pt wire, respectively. All electrode potentials are reported with respect to the RHE.

\section{Results}

3.1. Cyclic Voltammetry. Figure 1 shows cyclic voltammograms (CVs) of an $\mathrm{Au}(111)$ electrode in $0.1 \mathrm{M} \mathrm{HClO}_{4}$ (dotted line) and $0.1 \mathrm{M} \mathrm{HClO}_{4}+0.1 \mathrm{mM}$ TMA (solid line) using the so-called hanging meniscus method. Under these conditions, TMA is completely protonated $\left(\mathrm{p} K_{1}=2.1, \mathrm{p} K_{2}=4.1, \mathrm{p} K_{3}=\right.$ $5.18^{27}$ ). It can be seen that the double-layer region of the CV extends from 0 to $1.2 \mathrm{~V}$ in the absence of TMA. When the potential goes more positive, two well-separated oxidation peaks

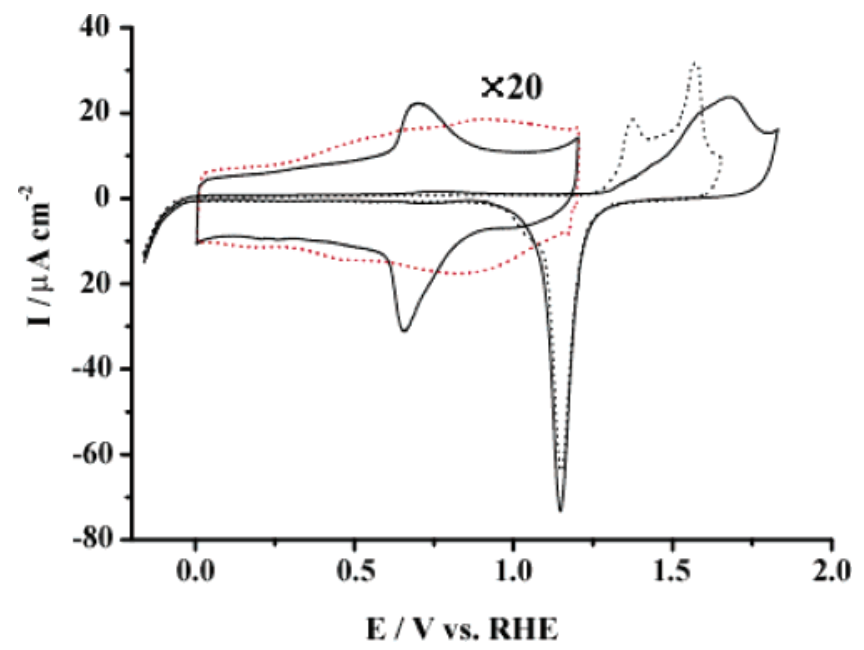

Figure 1. Cyclic voltammograms for $\mathrm{Au}(111)$ electrode in $0.1 \mathrm{M}$ $\mathrm{HClO}_{4}$ (dashed line) and $0.1 \mathrm{M} \mathrm{HClO}_{4}+0.1 \mathrm{mM}$ TMA (solid line). The scan rate was $50 \mathrm{mV} / \mathrm{s}$.

are observed, as described previously. ${ }^{28}$ It is indicated that the surface is well-defined $\mathrm{Au}(111)$ free from contamination.

After the examination of the bare $\mathrm{Au}(111)$ electrode, the electrochemical behavior of $\mathrm{Au}(111)$ is investigated in $0.1 \mathrm{M}$ $\mathrm{HClO}_{4}$ containing TMA shown in the solid line of Figure 1. The oxidation of the electrode surface in $0.1 \mathrm{mM}$ TMA is considerably hampered and shifted toward more positive potentials, which suggests strong adsorption of TMA molecules on the $\mathrm{Au}(111)$ surface. The result is similar to that of benzoic acid on a polycrystalline gold surface in $0.1 \mathrm{M} \mathrm{HClO}_{4}$ solution. ${ }^{19}$ Chemical structures show that the two molecules possess identical functional groups. Hence, it can be concluded that TMA molecules form a chemical bond to the surface as described by Zelenay et al. ${ }^{19}$

A pair of new peaks, ascribed to structural transformation in the TMA adlayer, is found in the double-layer region. With the increase of $\mathrm{pH}$, this transition region shifts toward more negative potentials. At $\mathrm{pH}>5$, no peaks are observed in the doublelayer region. The details on the transition with $\mathrm{pH}$ will be described in a separate paper. The observed feature of the $\mathrm{pH}$ dependence suggests that the structural transformation of the TMA adlayer may involve an interfacial protonation/deprotonation process. ${ }^{29}$ The main driving force may come from different electronic properties of the gold surface atom at different applied potentials. Namely, the applied potential can be exploited to drive the structural transition. The oxidation and reduction peaks were observed at almost the same potential 0.67 $\mathrm{V}$ and have the same charge of ca. $14.1 \mu \mathrm{C} \mathrm{cm}-2$, indicating that the protonation/deprotonation process is reversible. Similar results were also reported for fumaric acid on $\mathrm{Au}(111) .{ }^{29}$

3.2. STM Observation. A well-defined terrace-step structure is easily observed on the well-prepared $\mathrm{Au}(111)$ surface. The atomic image of $\mathrm{Au}(111)-(1 \times 1)$ is routinely discerned on the terrace in pure $\mathrm{HClO}_{4}$ solution. After the examination of the bare $\mathrm{Au}(111)$ surface, a small amount of TMA solution was directly added to an STM cell under the potential control of $0.65 \mathrm{~V}$. The concentration of TMA in $0.1 \mathrm{M} \mathrm{HClO}_{4}$ was ca. $0.1 \mathrm{mM}$. Half an hour later, a different pattern appears in the STM image, as shown in Figure 2a. It is evident that the atomically flat terraces are now covered by the ordered adlayers of TMA. The important feature in the image is triangular clusters, which coexist with elongated brighter spots.

Figure $2 b$ is a higher resolution STM image, revealing more details of the internal molecular structure, orientation, and 

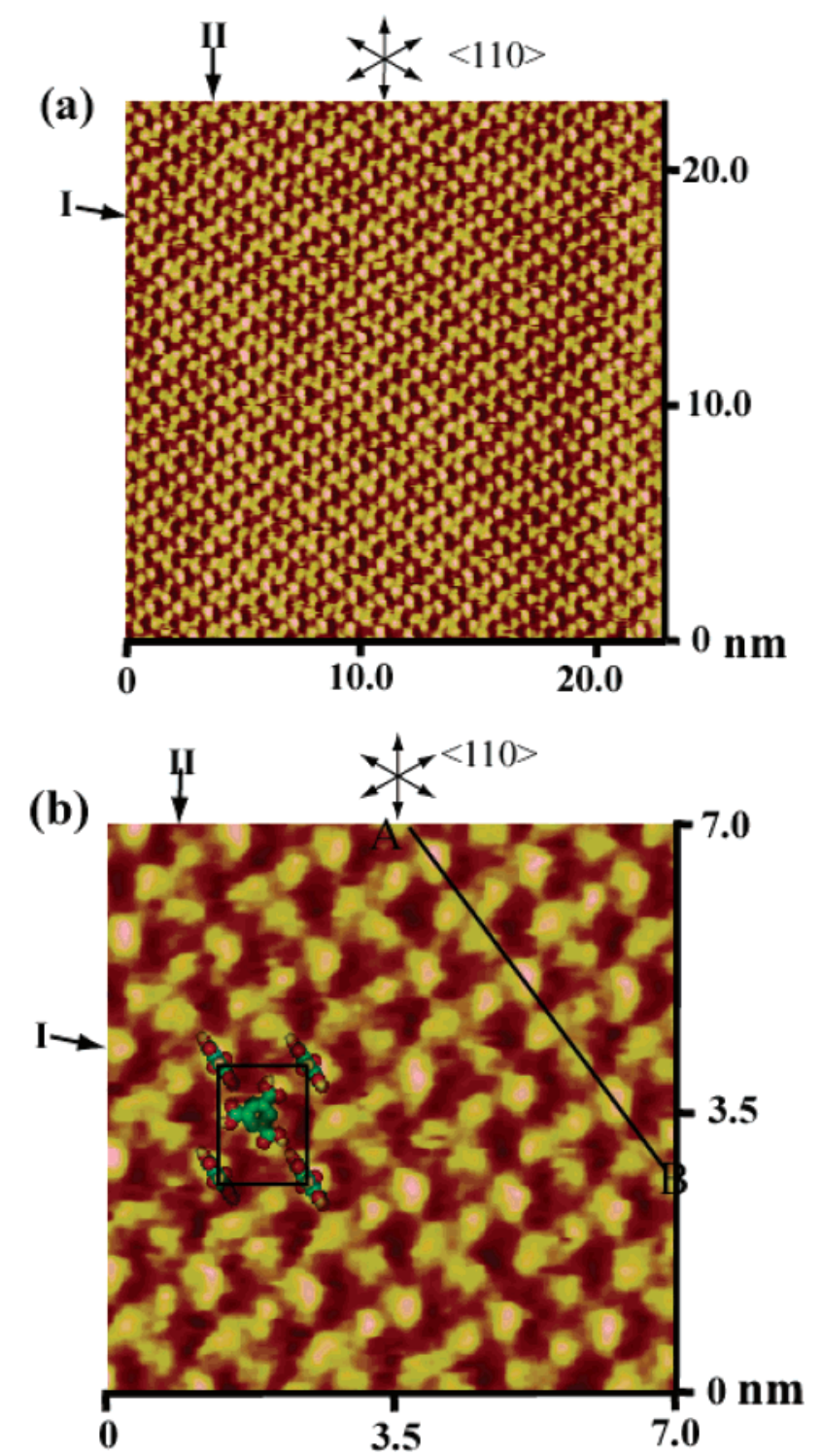

(c)

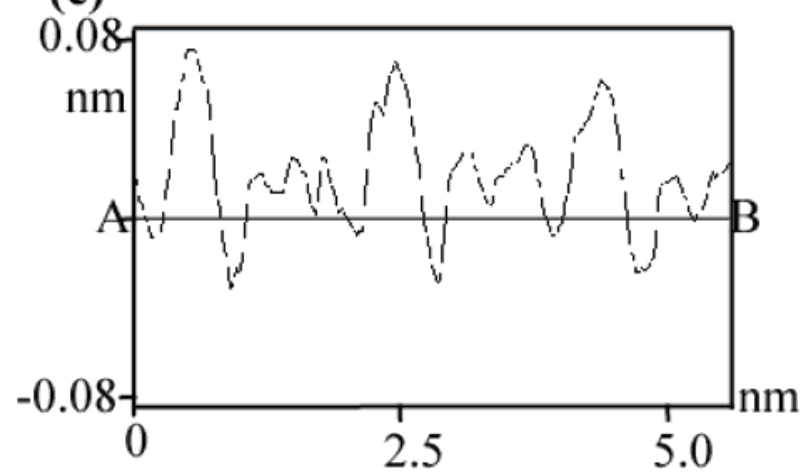

Figure 2. (a) Typical large scale STM image of the TMA adlayer on $\mathrm{Au}(111)$ in $0.1 \mathrm{M} \mathrm{HClO}_{4}$ containing $0.1 \mathrm{mM}$ TMA, obtained at $I_{t}$ (tunneling current) $=1.8 \mathrm{nA}$ and $E_{\mathrm{S}}$ (electrode potential of substrate) $=0.65 \mathrm{~V}$. (b) High-resolution STM image of TMA molecules adsorbed on $\mathrm{Au}(111)$ in $0.1 \mathrm{M} \mathrm{HClO}_{4}$ solution, obtained at $I_{\mathrm{t}}=2.7 \mathrm{nA}$ and $E_{\mathrm{s}}$ $=0.65 \mathrm{~V}$. A set of arrows indicates $\langle 110\rangle$ direction of $\mathrm{Au}(111)$. (c) Height profile along line $\mathrm{A}-\mathrm{B}$ revealing the different imaging contrast for adjacent TMA molecules and their intermolecular distances.

packing arrangement in the ordered adlayer. It can be seen that there are two different molecular rows marked by arrows I and II. In the row marked by II are sets of regular clusters. Each cluster appears as an equilateral triangle, which consists of three bright spots with similar corrugation of ca. $0.3 \AA$, as shown in
Figure 2c. It is indicated that the TMA molecule takes a flatlying orientation on the $\mathrm{Au}(111)$ surface with the phenyl ring parallel to the substrate. The result is favorable to that on $\mathrm{Cu}-$ (100) under UHV conditions. ${ }^{23,24}$ The symmetry and the characteristic side length of approximately $8 \AA$ agree well the with shape and dimensions of TMA. Therefore, each triangular cluster in the image can be assigned as one TMA molecule, and the bright spots can be attributed to the three "blades" of the carboxyl groups. The corrugation height along row I consisting of elongated bright spots is approximately $0.4 \AA$ higher than that in row II, as shown in the cross-sectional profile in Figure 2c. Compared with the triangular cluster, the individual bright spots may be ascribed to vertically oriented TMA molecules. On the basis of the above analysis, a rectangular unit cell is outlined in the STM image. Each unit cell includes four bright spots located in the four corners and a triangular cluster in the center. The characteristic molecular distances are $a=1.0 \pm 0.2 \mathrm{~nm}$ and $b=1.5 \pm 0.2 \mathrm{~nm}$, respectively. Namely, a $(5 \times 2 \sqrt{ } 3)$ structure is identified. Each unit cell is composed of two molecules, flat oriented and vertically oriented ones. The corresponding surface concentration is estimated to $2.3 \times 10^{-10}$ mol cm-2.

When the electrode potential is stepped in the negative direction, different STM images are observed, suggesting that local desorption takes place. However, one can still observe an ordered adlayer, which is rather fragile. Repetitive rapid scanning of the same electrode area disrupts the long range order of this phase. Parts $a$ and $b$ of Figure 3 show typical high-resolution STM images acquired at $0.25 \mathrm{~V}$. It is evident that the elongated spots found at positive potentials completely disappear and only triangular clusters are observed. These observations suggest that all TMA molecules are absorbed in a flat-lying orientation. The intermolecular distances along these rows are found to be $1.16 \pm 0.2 \mathrm{~nm}$, which corresponds to 4 times the lattice parameter of $\mathrm{Au}(111)$. Therefore, it can be concluded that TMA molecules form a $(4 \times 4)$ adlayer structure. A unit cell is indicated in Figure $3 \mathrm{~b}$. The corresponding surface concentration is estimated to be ca. $1.4 \times 10^{-10} \mathrm{~mol} \mathrm{~cm}{ }^{-2}$. Finally, we mention that complete desoprtion of TMA molecules takes place at $E<0.10 \mathrm{~V}$. The mixed adlayer structure in Figure 2 could be observed again when the electrode potential was stepped from 0.25 to $0.65 \mathrm{~V}$. It is suggested that the structural transformation is reversible with the applied electrode potential.

A new molecular structure appears at $E>0.80 \mathrm{~V}$, as shown in Figure 4. One can clearly observe an elongated striped structure along the $\langle 121\rangle$ crystallographic directions, which include three different molecular rows as marked by arrows I, II, and III. The disappearance of triangular clusters in the STM image suggests that no planar oriented TMA resides any more on $\mathrm{Au}(111)$. Careful observation reveals that there exist two different domains $\mathrm{A}$ and $\mathrm{B}$. The domain boundaries between $\mathrm{A}$ and $\mathrm{B}$ are indicated by dashed lines (Figure 4a). A highresolution image of the domain structure $\mathrm{A}$ is shown in Figure 4b. The topographic height of the molecular rows alternates: two adjacent rows are imaged more brightly; the third molecular row is slightly darker, which can be seen from the cross sectional profile of Figure $4 \mathrm{c}$. The cross-sectional profile along the $\mathrm{A}-\mathrm{B}$ direction shows different corrugation heights in the molecular rows. A ca. $0.4 \AA$ difference can be seen between the darker row III and brighter rows I and II, which does not reflect the geometric difference of the molecule. ${ }^{24}$ This is rationalized by the fact that the STM topography reflects electronic structure. Figure $4 \mathrm{~b}$ also shows the rhombohedral unit cell. The estimated molecular distances are $a=1.0 \pm 0.2 \mathrm{~nm}$ and $b=2.0 \pm 0.2$ 
(a)

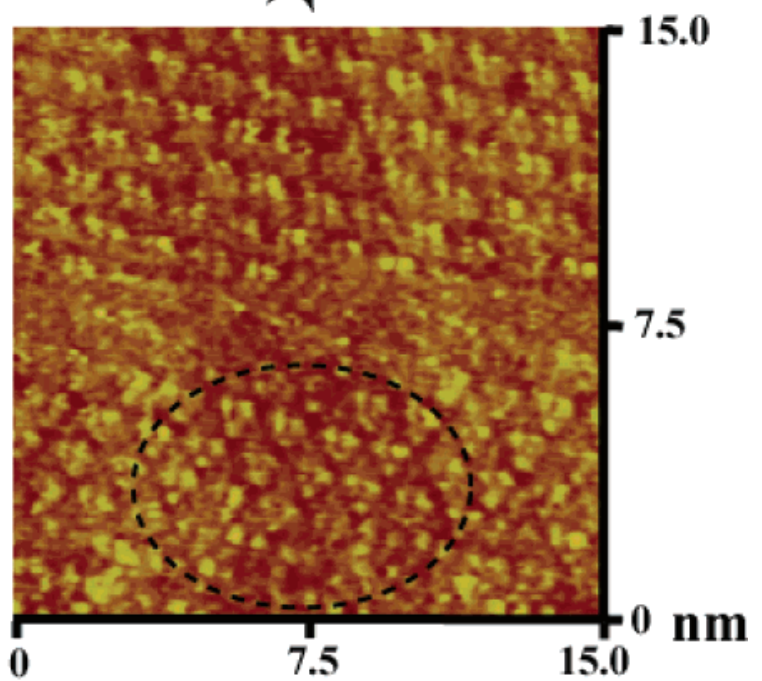

(b)

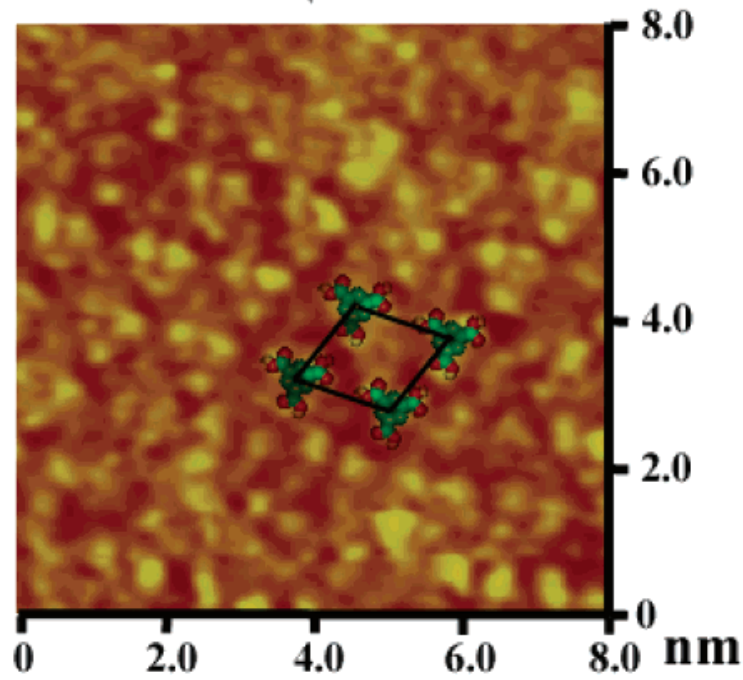

Figure 3. (a) High-resolution STM image of the TMA adlayer on $\mathrm{Au}(111)$ in $0.1 \mathrm{M} \mathrm{HClO}_{4}$ containing $0.1 \mathrm{mM}$ TMA. The image was obtained at $I_{\mathrm{t}}=612 \mathrm{pA}$ and $E_{\mathrm{s}}=0.25 \mathrm{~V}$. (b) Magnification of the framed area in (a). A set of arrows indicates $\langle 110\rangle$ direction of $\mathrm{Au}-$ (111).

$\mathrm{nm}$, respectively. Compared with the underlying $\mathrm{Au}(111)$ lattice, a $(2 \sqrt{3} \times 4 \sqrt{3})$ structure can be identified, which gives rise to a local surface concentration of $2.9 \times 10^{-10} \mathrm{~mol} \mathrm{~cm}^{-2}$. Similar observations revealed that the corresponding unit cell in domain B is approximated by a $(7 \times 2 \sqrt{ } 3)$ structure.

\section{Discussion}

The potential dependent STM experiments in $0.1 \mathrm{M} \mathrm{HClO}_{4}$ demonstrate that TMA adsorbs on $\mathrm{Au}(111)$ and forms three well-defined adlayers. When the electrode potential is stepped to more positive, the surface concentration of TMA molecules increases from $1.4 \times 10^{-10}$ to $2.9 \times 10^{-10} \mathrm{~mol} \mathrm{~cm}^{-2}$. The reversible peaks at around $0.67 \mathrm{~V}$ in the $\mathrm{CV}$ correspond to a phase transition within the molecular adlayer. From the chemical structure, the TMA molecule is composed of three carboxylate groups and one phenyl ring. The molecule can interact with the $\mathrm{Au}$ surface either via the lone electron pairs of the deprotonated carboxylate groups (tilted or perpendicular orienta- (a)

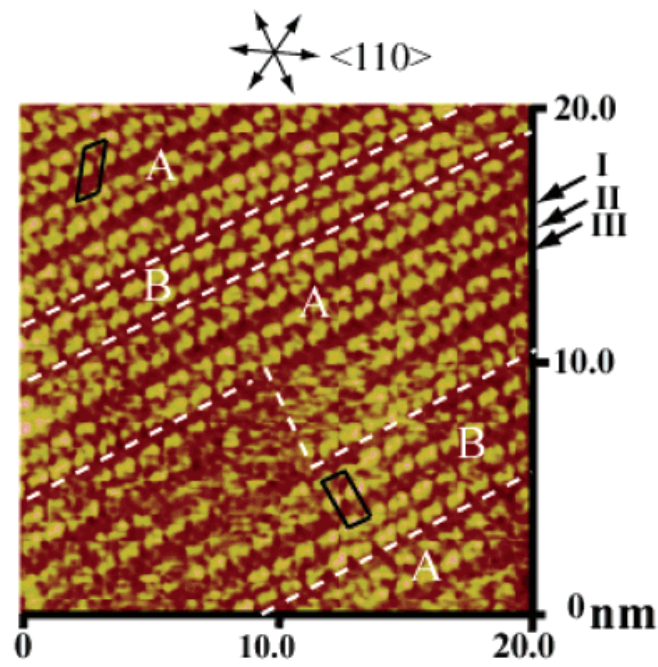

(b)

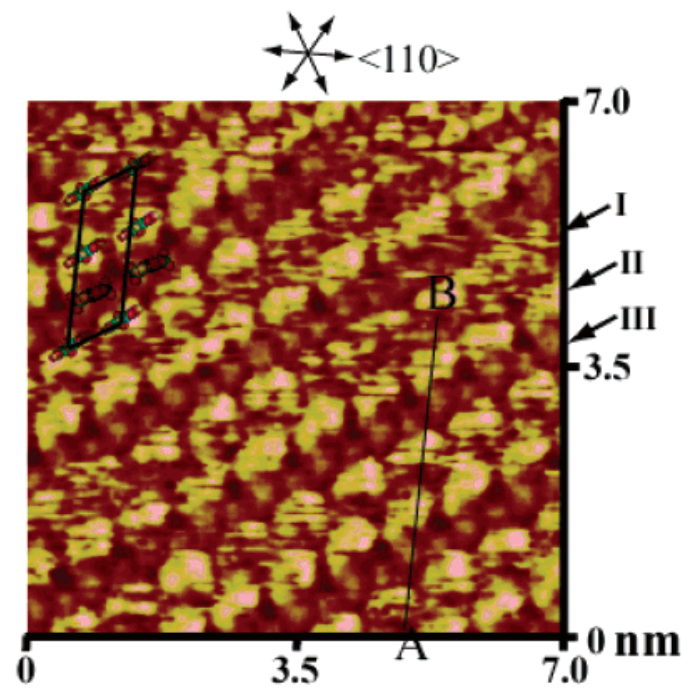

(c)

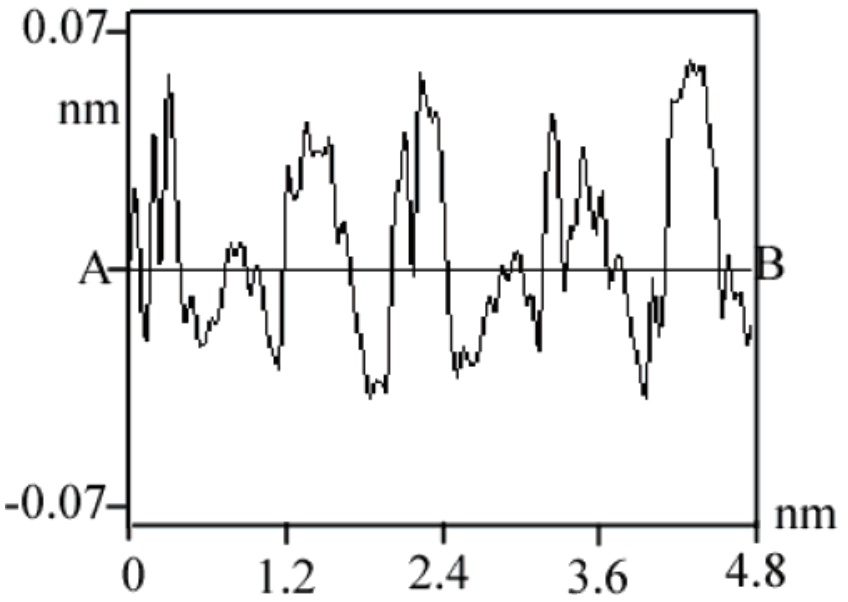

Figure 4. (a) Typical large scale STM image of the TMA adlayer on $\mathrm{Au}(111)$ in $0.1 \mathrm{M} \mathrm{HClO}_{4}$ containing $0.1 \mathrm{mM}$ TMA, obtained at $I_{\mathrm{t}}=$ $1.4 \mathrm{nA}$ and $E_{\mathrm{s}}=0.85 \mathrm{~V}$. (b) High-resolution STM image of TMA adlayers on $\mathrm{Au}(111)$ in $0.1 \mathrm{M} \mathrm{HClO}_{4}$ containing $0.1 \mathrm{mM}$ TMA, obtained at $I_{\mathrm{t}}=1.4 \mathrm{nA}$ and $E_{\mathrm{s}}=0.85 \mathrm{~V}$. A set of arrows indicates $\langle 110\rangle$ direction of $\mathrm{Au}(111)$. (c) Height profile along line $\mathrm{A}-\mathrm{B}$ revealing the different imaging contrast for adjacent TMA molecules and their intermolecular distances.

tion) or with the $\pi$-electrons of the aromatic ring (planar orientation). As reported previously, benzoic acid, which has one carboxyl group, adsorbs on the Au surface flat at negative 

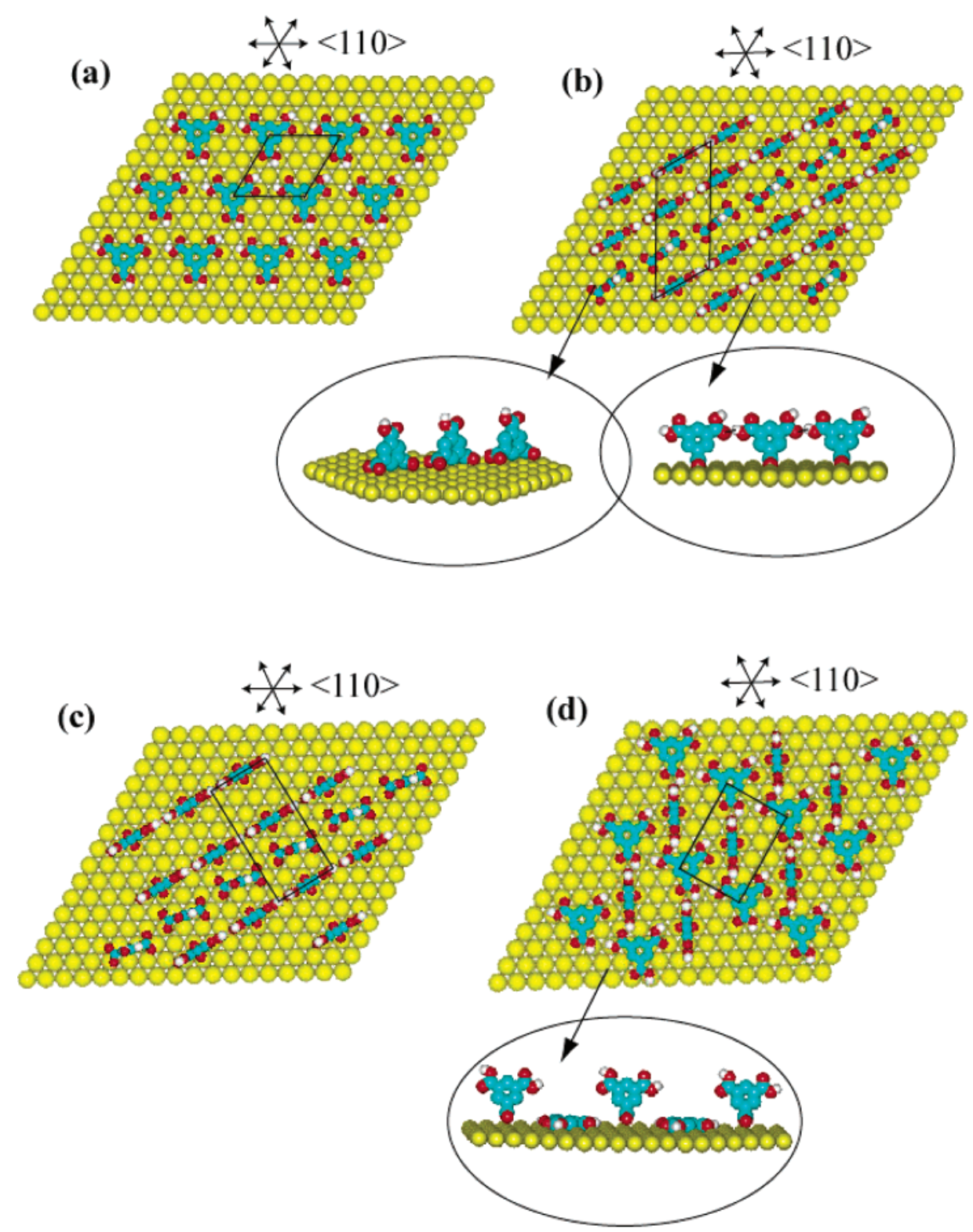

Figure 5. Schematic representation of the suggested TMA adlayer structures on Au(111). Model (a) corresponds to the STM images in Figure 3 , (b) and (c) to the domain A and B of the STM images in Figure 4, and (d) to the STM images in Figure 2. A set of arrows indicates $\langle 110\rangle$ direction of $\mathrm{Au}(111)$.

charge densities and in a vertical orientation at positive charge densities, respectively. ${ }^{19}$ On the basis of the molecular structure of TMA and a careful analysis of the STM experiments, we propose the following structural models (Figure 5).

Figure 5a represents the $(4 \times 4)$ adlayer, which corresponds to the STM images acquired at $0.25 \mathrm{~V}$ in Figure 3. We suggest flat lying TMA molecules supported on the Au(111) surface by a strong $\pi$ electron interaction with the substrate. The TMA molecules are tentatively positioned at 3-fold hollow sites, similar to benzene on $\mathrm{Cu}(111), \operatorname{Pt}(111)$, and $\mathrm{Rh}(111){ }^{30,31}$ Previous studies show that TMA molecules can form a honeycomb supramolecular network structure when flatly absorbed on the $\mathrm{Cu}(100)$ surface or in the crystal structure of bulk $\alpha$-polymorph TMA reported by Kern et al. ${ }^{24}$ The main driving force for the formation of such a structure can be attributed to the intermolecular hydrogen bonds between the carboxyl groups of TMA molecules. In contrast, the employment of $\mathrm{Au}(111)$ not only changes the molecule-substrate interaction but also the commensurate between the molecule and the lattice parameter of gold. Meanwhile, the present experiment is conducted under aqueous conditions. The water may play an important role in forming ordered adlayers of TMA molecules. Thus, different supramolecular conformations have been observed.

Figure 5b shows the proposed structural model for domain A of Figure 4 with a $(2 \sqrt{ } 3 \times 4 \sqrt{ } 3)$ structure. On the basis of the knowledge how different functional groups interact with surfaces, ${ }^{24,32}$ it is suggested that the TMA molecule reorients at positive charge densities from a flat-lying to an upright orientation. The deprotonated carboxylate faces toward the surface and free electron pairs on oxygen are coordinated by the positively charged surface, which reflects the formation of a distinct chemisorption bond similar to TMA on $\mathrm{Cu}(100){ }^{24}$ Both calculation $^{33,34}$ and experimental evidence ${ }^{35,36}$ indicate that uprightly oriented carboxylate groups have a strong preference toward the coordination of both oxygen atoms in 2-fold bridge adsorption sites on the electrode surface. The different corrugation heights of the experimentally observed molecular rows are probably related to different bonding configurations of TMA molecules at the substrate, possibly through either one or two 
deprotonated carboxyl groups resulting in different conductivities of the molecule on the surface. ${ }^{24}$ Our tentative adsorption scenario, based on geometrical reasoning, strongly favors a structural model of bidentate gold-carboxylate entities located at a short bridge site, or through two carboxylates, both centered at the short-bridge site and thus rotated with respect to the plane of the phenyl ring by $90^{\circ}$, with the molecule's center at a hollow site. ${ }^{24,37}$ For TMA adsorbed at short bridges in rows I and II, we make use of the fact that carboxylates can freely rotate around the $\mathrm{C}-\mathrm{C}$ bond. ${ }^{24,38}$ The rotation of the molecular plane is attributed to intermolecular interactions to optimize the hydrogen-bonding configuration. This arrangement, illustrated in Figure $5 b$, is believed to be stabilized by $\mathrm{H}$-bond formation with an $\mathrm{OH}-\mathrm{-O}$ bond length of $1.9 \AA$ between the undissociated carboxyl groups in the first and in the second rows along the $\langle 121\rangle$ direction. A similar arrangement of molecules within rows via hydrogen-bonded species was also proposed for tartaric acid on $\mathrm{Cu}(110) .{ }^{39}$ The third row, where STM revealed periodic changes in the imaging height, is believed to represent TMA molecules coordinated with two carboxylate groups to the gold surface with the phenyl ring centered at a hollow site. Apparently, the different contrast in the STM images for different rows comes from different conductivity of the molecule depending on its orientation on the surface. TMA with one carboxyl group coordinated to the electrode surface has better conductivity in comparison with those with two carboxyl groups coordinated. Furthermore, TMA molecules in short bridge sites give a brighter contrast than those adsorbed in hollow sites. ${ }^{24}$ On the basis of similar arguments, we also suggest a structural model of domain $\mathrm{B}$ in the high-coverage phase (Figure $5 \mathrm{c}$ ). In the intermediate potential region (Figure 2), the TMA molecules coexist in a mixed planar and perpendicular orientation, giving rise to a $(5 \times 2 \sqrt{ } 3)$ structure. The structural model of this adlayer is shown in Figure 5d. Four molecules assuming a vertical orientation are located in corner positions of the unit cell with one carboxylate group coordinated to a short bridge site. The phenyl ring is assumed to be rotated by $90^{\circ}$ due to intermolecular repulsion. ${ }^{38}$ The center of the unit cell contains a flat-lying molecule. According to the structural models, it can be concluded that the distances and angle bear a simple relationship to the corresponding substrate vector within the experimental error in the present study. All the ordered adlayers of TMA obtained at different electrode potential are commensurate with respect to the $\mathrm{Au}(111)$ lattice. It is suggested that the moleculesubstrate interactions plays an important role in forming ordered TMA adlayers. Changing molecule-substrate interactions by applying different electrode potentials may result in structural transformation.

The above adlayer structures and phase transition of TMA on $\mathrm{Au}(111)$ are significantly different from that of TMA adsorbed on $\mathrm{Cu}(100)$ reported by Kern et al. ${ }^{24}$ In general, the formation of ordered adlayer is dominated by the intermolecular and molecule-substrate interactions. The different electronic properties of substrates used in the two systems are thought to play an important role in the adlayer structure and orientation of TMA on surface. The different thermodynamic and kinetic behaviors of two phases of TMA on $\mathrm{Cu}(100)$ account for the irreversible phase transformation observed by Kern et al. ${ }^{24}$ In contrast, the present study demonstrates that the potential dependent phase transition of TMA on $\mathrm{Au}(111)$ is reversible. However, the exact role of surface electronic properties, the nature of external stimuli applied in the phase transition, could not be simply concluded from the present results. It is evident that more straightforward experimental techniques such as IR and theoretical calculations must be applied to obtain further structural information to support the above results and obtain insight into the underlying essence of the phase transition. A comprehensive study of SEIRAS combined with an attenuated total reflection (ATR) is now in preparation and will be reported in a forthcoming contribution. ${ }^{40}$

\section{Conclusion}

In situ STM and cyclic voltammetry have been employed to investigate the adsorption of TMA molecules on $\mathrm{Au}(111)$ in aqueous electrolytes. High-resolution STM images reveal the internal structure and orientation of TMA molecules at different potentials. In $0.1 \mathrm{M} \mathrm{HClO}_{4}$, the adlayer structures are potential dependent. A pair of reversible peaks, ascribed to an adlayer phase transition, appears in the $\mathrm{CV}$ at $0.67 \mathrm{~V}$. At negative potentials, such as $0.25 \mathrm{~V}$, flat lying TMA molecules form a $(4 \times 4)$ structure. At positive potentials, such as $0.85 \mathrm{~V}$, TMA molecules are vertically adsorbed on the $\mathrm{Au}(111)$ surface and form a $(2 \sqrt{3} \times 4 \sqrt{ } 3)$ adlayer structure. In the intermediate potential region, a $(5 \times 2 \sqrt{ } 3)$ structure with coexisting flat-lying and vertical orientated molecules was found. Three structural models are proposed for the three adlayers. All these results indicate that the phase transition is governed entirely by the applied electrical field.

Acknowledgment. Financial supports from National Natural Science Foundation of China (Nos. 20025308, 20177025, and 20103008), National Key Project on Basic Research (Grant G2000077501) and the Chinese Academy of Sciences are gratefully acknowledged. T.W. acknowledges support of the Volkswagen Foundation (I 75-262) and of the Research Center Jülich.

\section{References and Notes}

(1) Hubbard, A. T. Chem. Rev. 1988, 88, 633.

(2) Lipkowski, J., Ross, P. N., Eds. Adsorption of Molecules at Metal Electrodes; VCH Publisher: New York, 1992.

(3) Soriaga, M. P. Prog. Surf. Sci. 1992, 39, 325.

(4) Hagenström, H.; Schneeweiss, M. A.; Kolb, D. M. Langmuir 1999, $15,2435$.

(5) Esplandiú, M. J.; Hagenström, H.; Kolb, D. M. Langmuir 2001 , 17,828 .

(6) Wandlowski, Th. In Encyclopedia of Electrodhemistry; Gileadi, E., Urbakh, M., Eds.; Vo1. 1, Chapter 3.3.

(7) Itaya, K. Prog. Surf. Sci. 1998, 58, 121.

(8) Ohira, A.; Ishizaki, T.; Sakata, M.; Kunitake, M. J. Electroanal. Chem. 1999, 472, 163.

(9) Giz, M. J.; Duong, B.; Tao, N. J. J. Electroanal. Chem. 1999, 465, 72

(10) Wan, L. J.; Wang, C.; Bai, C. L.; Osawa, M. J. Phys. Chem. B 2001, 105, 8399.

(11) Wan, L. J.; Noda, H.; Hara, Y.; Osawa, M. J. Electroanal. Chem. 2000, 489, 68 .

(12) Noda, H.; Wan, L. J.; Osawa, M. Phys. Chem. Chem. Phys. 2001, 3,3336 .

(13) Wandlowski, Th. J. Electroanal. Chem. 1995, 395, 83.

(14) Wandlowski, Th.; Hölzle, M. H. Langmuir 1996, 12, 6604.

(15) Hölzle, M. H.; Wandlowski, Th.; Kolb, D. M. J. Electroanal. Chem 1995, 394, 271.

(16) Cunha, F.; Tao, N. J.; Wang, X. W.; Jin, Q.; Duong B.; D’Agnese, J. Langmuirr 1996, 12, 6410.

(17) Wan, L. J.; Noda, H.; Wang C., Bai C. L.; Osawa, M. ChemPhysChem 2001, 1439 .

(18) Cai, W. B.; Wan, L. J.; Osawa, M. Langmuir 1998, 14, 6992.

(19) Zelenay, P.; Waszczuk, P.; Dobrowolska, K.; Sobkowski, J. Electrochim. Acta 1994, 39, 655.

(20) Kolotuchin, S. V.; Thiessen, P. A.; Fenlon, E. E.; Wilson, S. R.; Loweth, C. J.; Zimmerman, S. C. Chem. Eur. J. 1999, 5, 2537.

(21) Griessl, S.; Lackinger, M.; Edelwirth, M.; Hietschold, M.; Heckl, W. M. Single Mol. 2002, 1, 25.

(22) Messina, P.; Dmitriev, A.; Lin, N.; Spillmann, H.; Abel, M.; Barth, J. V.; Kern, K. J. Am. Chem. Soc. 2002, 124, 14000. 
(23) Lin, N.; Dmitriev, A.; Weckesser, J.; Barth, J. V.; Kern, K. Angew. Chem., Int. Ed. 2002, 41, 4779.

(24) Dmitriev, A.; Lin, N.; Weckesser, J.; Barth, J. V.; Kern, K. J. Phys. Chem. B 2002, 106, 6907.

(25) Ishikawa, Y.; Ohira, A.; Sakata, M.; Hirayama, C.; Kunitake, M. Chem. Commun. 2002, 2652.

(26) Wan, L. J.; Shundo, S.; Inukai, J.; Itaya, K. Langmuir 2000, 16, 2164.

(27) Dean, J. A. Lange's Handbook of Chemistry, 13th ed.; McGrawHill Book Co.: New York, 1985; pp 5-42.

(28) Angerstein-Kozlowska, H.; Conway, B. E.; Hamelin, A.; Stoicoviciu, L. J. Electroanal. Chem. 1987, 228, 429.

(29) Noda, H.; Ataka, K.; Wan, L. J.; Osawa, M. Surf. Sci. 1999, 427$428,190$.

(30) Wan, L. J.; Itaya, K. Langmuir 1997, 13, 7173.

(31) Yau, S. L.; Kim, Y. G.; Itaya, K. J. Am. Chem. Soc. 1996, 118, 7795.

(32) Chen, Q.; Perry, C. C.; Frederik, B. G.; Murray, P. W.; Haq, S.; Richardson, N. V. Surf. Sci. 2000, 446, 63.
(33) Casarin, M.; Granozzi, G.; Sambi, M.; Tondello, E. Surf. Sci. 1994, 307-309, 95.

(34) Sambi, M.; Granozzi, G.; Casarin, M.; Rizzi, G. A.; Vittadini, A.; Caputi, L. S.; Chiarello, G.; Surf. Sci. 1994, 315, 309.

(35) Weiss, K. U.; Dippel, R.; Schindler, K. M.; Gardner, P.; Fritzsche, V.; Bradshaw, A. M. Phys. Rev. Lett. 1992, 69, 3196.

(36) Woodruff, D. P.; McConville, C. F.; Kilcoyne, A. L. D. Surf. Sci. 1988, 201, 228.

(37) Haq, S.; Bainbridge, R. C.; Frederick, B. G.; Richardson, N. V. J. Phys. Chem. B 1998, 102, 8807.

(38) Frederick, B. G.; Chen, Q.; Barlow, S. M.; Condon, N. G.; Leibsle, F. M.; Richardson, N. V. Surf. Sci. 1996, 352-354, 238.

(39) Lorenzo, M. O.; Baddeley, C. J.; Muryn, C.; Raval, R. Nature 2000, 404, 376.

(40) Han, B.; Pronkin, S.; Su, G. J.; Wan, L. J.; Wandlowski, Th. Manuscript in preparation. 